Publ. RIMS, Kyoto Univ.

15 (1979), 749-766

\title{
Time Decay and Scattering for Some Nonlinear Wave Equation
}

\author{
By \\ Takao KaKita, ${ }^{*}$ Kenji NishIHARA** and Chiharu TAMAmURA***
}

\section{§1. Introduction}

Recently J. M. Chadam proved a global existence and uniqueness theorem to the Cauchy problem for nonlinear Klein-Gordon equations (in three dimensional space) with nonlinear term $G\left(x, t, u, u_{x}, u_{t}\right)$ [1] and discussed also scattering theory for them [2].

The scattering theory for those equations have been studied by [4], [5], [9], [10], [11], [12], etc. On the other hand, M. Reed in his lecture notes [8] developed an abstract theory of global existence-uniqueness and scattering theory for nonlinear wave equations having a nonlinear Klein-Gordon equation as a specific example

$$
\square u+m^{2} u=-u^{p}, \quad x \in \mathbb{R}^{s}
$$

in which we are interested.

Our purpose is to prove an abstract existence-uniqueness theorem and to discuss scattering theory for nonlinear wave equations, by extending the method in [8] so that equations of the form

$$
\square u+m^{2} u+g_{1} u^{\beta}+g_{2} u_{t}^{r}=0
$$

are included as examples.

First we introduce some notations. Let $\mathscr{C}$ be a Hilbert space with norm $\|\cdot\|_{\mathscr{C}}=\|\cdot\|$ and let the following auxiliary norms on $\mathscr{H}$ be given: for each $j=1,2\|\cdot\|_{a_{j}}$ satisfies all the norm conditions except that $\|\phi\|_{a_{j}}=0$ implies $\phi=0$, and $\|\cdot\|_{b_{j}}$ satisfies all the norm conditions except that it

Communicated by S. Matsuura, August 19, 1977.

* Department of Mathematics, School of Science and Engineering. Waseda University.

** Nerima Senior High School, Tokyo.

*** Statistics Division, Institute of Developing Economies, Tokyo. 
may take the value $+\infty$. Let $A$ be a self-adjoint operator on $\mathscr{H}$ and $J_{j}$ be nonlinear mappings: $\mathscr{H} \rightarrow \mathcal{H}$ which satisfy the following hypotheses: for $\phi \in \mathscr{H}$ and each $j=1,2$,

(H1) there is a positive constant $C$ such that

$$
\|\phi\|_{a_{j}} \leqq C\|\phi\|,
$$

(H2) there are positive constants $C$ and $d_{j}$ such that

$$
\left\|e^{-i A t} \phi\right\|_{a_{j}} \leqq C|t|^{-d_{j}}\|\phi\|_{b_{j}} \quad \text { when }|t| \geqq 1
$$

and

(H3) there exist positive constants $h_{j}, \delta, p$ (with $p \geqq 1, d_{1} p>1$ ) and $q$ (with $q \geqq 1, d_{2} q>1$ ) so that for all $\phi, \psi \in \mathcal{H}$ satisfying $\|\phi\| \leqq \delta,\|\psi\| \leqq \delta$, there hold

$$
\begin{aligned}
& \left\|J_{1}(\phi)-J_{1}(\psi)\right\| \leqq h_{1} \sum_{\varepsilon_{1}}\left(\|\phi\|_{a_{1}}+\|\psi\|_{a_{1}}\right)^{p-\varepsilon_{1}}\|\phi-\psi\|_{a_{1}}^{\varepsilon_{1}}\|\phi-\psi\|^{1-\varepsilon_{1}}, \\
& \left\|J_{2}(\phi)-J_{2}(\phi)\right\| \leqq h_{2} \sum_{\varepsilon_{2}}\left(\|\phi\|_{a_{2}}+\|\psi\|_{a_{2}}\right)^{q-\varepsilon_{2}}\|\phi-\psi\|_{a_{2}}^{\varepsilon_{2}}\|\phi-\psi\|^{1-\varepsilon_{2}}, \\
& \left\|J_{1}(\phi)-J_{1}(\psi)\right\|_{b_{j}} \leqq h_{1} \sum_{\sigma_{j}}\left(\|\phi\|_{a_{1}}+\|\psi\|_{a_{1}}\right)^{p-\sigma_{j}}\|\phi-\psi\|_{a_{1}}^{\sigma_{j}}\|\phi-\psi\|^{1-\sigma_{j}}
\end{aligned}
$$

and

$$
\left\|J_{2}(\phi)-J_{2}(\psi)\right\|_{b_{j}} \leqq h_{2} \sum_{\rho_{j}}\left(\|\phi\|_{a_{2}}+\|\psi\|_{a_{2}}\right)^{q-\rho_{j}}\|\phi-\psi\|\left\|_{a_{2}}^{\rho_{j}}\right\| \phi-\psi \|^{1-\rho_{j}}
$$

where $\varepsilon_{j}, \sigma_{j}$ and $\rho_{j}$ run through some finite sets in $[0,1]$. Here in case $p=1$ or $q=1$, we assume each $h_{j}$ can be chosen arbitrarily small if $\delta$ is chosen small.

Now we define the scattering states $\Sigma_{\text {scat }}$ with norm $\|\cdot\|_{\text {scat }}$ by

$$
\Sigma_{\text {scat }}=\left\{\phi \in \mathcal{F} ;\|\phi\|_{\text {scat }}<\infty\right\}
$$

where

$$
\begin{aligned}
& \|\phi\|_{\text {scat }}=\left\|e^{-i t A} \phi\right\|, \quad\|\cdot\| \quad \text { being defined by } \\
& \|\phi\|=\sup _{\boldsymbol{R}}\left(\|\psi(r)\|+\sum_{j=1}^{2}(1+|r|)^{d_{j}}\|\psi(r)\|_{a_{j}}\right)
\end{aligned}
$$

for $\psi(\boldsymbol{r})$, a $\mathcal{K}$-valued function on $\boldsymbol{R}$. We observe that for all $t \in \mathbb{R}$

$$
\left\|e^{-i t A} \phi\right\|_{a_{j}} \leqq C(1+|t|)^{-d_{j}}\left(\|\phi\|+\|\phi\|_{b_{j}}\right),
$$

if $\|\phi\|_{b_{j}}<\infty$ with $j=1,2$. For simplicity we often use 
$p_{j}$ as $p_{1}=p$ and $p_{2}=q ; w_{j}$ as $w_{1}=\sigma_{1}$ and $w_{2}=\rho_{1}$; and

$z_{j}$ as $z_{1}=\sigma_{2}$ and $z_{2}=\rho_{2}$.

\section{$\S$ 2. Abstract Theorems}

The following lemma will be frequently used.

Lemma 2. 1 ([10], [8]). (a) If $a, b>0$ and $\max (a, b)>1$, then

$$
\int_{-\infty}^{\infty}(1+|t-s|)^{-a}(1+|s|)^{-b} d s \leqq C(1+|t|)^{-\min (a, b)}
$$

(b) If $a, b>0$ and $\max (a, b)>\min (a, b)$, then

$$
\sup _{\boldsymbol{R}}(1+|r|)^{\min (a, b)} \int_{t_{1}}^{t_{2}}(1+|r-s|)^{-a}(1+|s|)^{-b} d s
$$

tends to 0 as $t_{1}, t_{2} \rightarrow+\infty$ (or $-\infty$ ).

Our first main theorem is

Theorem 2.2 (global existence and uniqueness for small data). Assume $A, J_{j}$ and the norms $\|\cdot\|,\|\cdot\|_{a_{j}},\|\cdot\|_{b_{j}}$ satisfy $(\mathrm{H} 1),(\mathrm{H} 2)$ and (H3) in $\S 1$, and let $J=J_{1}+J_{2}$. Then there exists an $\eta_{0}>0$ such that for all $\phi_{-} \in \Sigma_{\text {scat }}$ with $\left\|\phi_{-}\right\|_{\text {scat }} \leqq \eta_{0}$, the integral equation

$$
\phi(t)=e^{-i t A} \phi_{-}+\int_{-\infty}^{t} e^{-i A(t-s)} J(\phi(s)) d s
$$

has a solution in $C((-\infty, \infty) ; \mathcal{H})$ with $\|\phi(\cdot)\| \leqq 2 \eta_{0}$, and the solution is unique when $p, q>1, d_{1} p>d_{2}$ and $d_{2} q>d_{1}$.

Proof. (I) Existence. As our method is based on the contraction mapping principle, we introduce a complete metric space $X\left(\eta, \phi_{-}\right)$with norm $\|\cdot\| \|$ defined by

$$
X\left(\eta, \phi_{-}\right)=\left\{\psi(t) ; \psi(t) \in C((-\infty, \infty) ; \mathcal{H}) \text { and }\left\|\psi-e^{-i t A} \phi_{-}\right\| \leqq \eta\right\}
$$

where $\left\|\phi_{-}\right\|_{\text {scat }} \leqq \eta \leqq \delta, \delta$ being a constant in (H3). Let $g$ be an operator on $X\left(\eta, \phi_{-}\right)$given by 


$$
(g \psi)(t)=\int_{-\infty}^{t} e^{-i A(t-s)} J(\psi(s)) d s \text { for } \quad \phi(\cdot) \in X\left(\eta, \phi_{-}\right) .
$$

It can easily be seen that $e^{-i A(t-s)} J(\psi(s))$ is a continuous function of $s$ for each fixed $t$ and that

$$
\|\psi\| \leqq\left\|e^{i A t} \phi_{-}\right\|+\eta \leqq 2 \eta .
$$

Under the hypotheses (H1), (H2) and (H3) we have the following estimates.

$$
\begin{aligned}
\left\|J_{j}(\psi(s))\right\| & \leqq \sum_{\varepsilon_{j}} h_{j}\|\psi(s)\|_{a_{j}}^{p_{j}}\|\psi(s)\|^{1-\varepsilon_{j}} \\
& \leqq \sum_{\varepsilon_{j}} h_{j}(2 \eta)^{p_{j}+1-\varepsilon_{j}}(1+|s|)^{-d_{j} p_{j}} \text { for } j=1,2 .
\end{aligned}
$$

and (since $d_{j} p_{j}>1$ )

$$
\|(g \psi)(t)\| \leqq \int_{-\infty}^{\infty}\|J(\psi(s))\| d s \leqq c_{0}\left(h_{1}, h_{2}, \eta\right)<+\infty
$$

$\|(g \psi)(t)\|_{a_{1}} \leqq \int_{-\infty}^{\infty}\left\|e^{-i A(t-s)} J(\psi(s))\right\|_{a_{1}} d s$

$$
\leqq c_{1}\left(h_{1}, h_{2}, \eta\right)(1+|t|)^{-d_{1}} \quad \text { if } \quad d_{2} p_{2} \geqq d_{1}
$$

$$
\begin{aligned}
\|(g \psi)(t)\|_{a_{2}} & \leqq \int_{-\infty}^{\infty}\left\|e^{-i A(t-s)} J(\psi(s))\right\|_{a_{2}} d s \\
& \leqq c_{2}\left(h_{1}, h_{2}, \eta\right)(1+|t|)^{-d_{2}} \quad \text { if } \quad d_{1} p_{1} \geqq d_{2} .
\end{aligned}
$$

Here we remark that all the constants $c_{j}\left(h_{1}, h_{2}, \eta\right)$ depend on $h_{1}, h_{2}$ and $\eta$ so that $c_{j}$ 's tend to 0 when $h_{1}, h_{2} \rightarrow 0$ or $\eta \rightarrow 0$. In fact, when $d_{2} p_{2} \geqq d_{1}$

$$
\begin{gathered}
\int_{-\infty}^{\infty}\left\|e^{-i A(t-s)} J(\psi(s))\right\|_{a_{1}} d s \\
\leqq c \int_{-\infty}^{\infty}(1+|t-s|)^{-d_{1}}\left(\|J(\psi(s))\|+\|J(\psi(s))\|_{b_{1}}\right) d s \\
\leqq c \sum_{j=1}^{2} h_{j} \int_{-\infty}^{\infty}(1+|t-s|)^{-d_{1}}\|\psi(s)\|_{a_{j}}^{p_{j}} \\
\quad \times\left(1+\sum_{\varepsilon_{j}}\|\psi(s)\|^{1-\varepsilon_{j}}+\sum_{w_{j}}\|\psi(s)\|^{1-w_{j}}\right) d s \\
\leqq c \sum_{j=1}^{2} h_{j}(2 \eta)^{p_{j}}\left(1+\sum_{\varepsilon_{j}}(2 \eta)^{1-\varepsilon_{j}}+\sum_{w_{j}}(2 \eta)^{1-w_{j}}\right) \\
\times \int_{-\infty}^{\infty}(1+|t-s|)^{-d_{1}}(1+|s|)^{-d_{j} p_{j}} d s
\end{gathered}
$$




$$
\leqq c_{2}\left(h_{1}, h_{2}, \eta\right)(1+|t|)^{-d_{1}} .
$$

For the inequality (4), a required estimate can be done analogously. It follows $\|\mathscr{g} \psi\|<\infty$ from (2), (3), (4).

Now we define a mapping $M: X\left(\eta, \phi_{-}\right) \rightarrow X\left(\eta, \phi_{-}\right)$by

$$
(M \psi)(t)=e^{-i A t} \phi_{-}+(\mathscr{g} \psi)(t) .
$$

We want to show that $M$ is a contraction mapping on $X\left(\eta, \phi_{-}\right)$provided $\eta$ is taken to be sufficiently small. Similar calculations to (2), (3), (4) lead to the inequalities:

$$
\begin{aligned}
& \|(g \phi)(t)-(\mathscr{g} \psi)(t)\| \leqq c_{0}^{\prime}\left(h_{1}, h_{2}, \eta\right)\|\phi-\psi\| \\
& \|(g \phi)(t)-(\mathscr{g} \psi)(t)\|_{a_{1}} \leqq(1+|t|)^{-d_{1}} c_{1}^{\prime}\left(h_{1}, h_{2}, \eta\right)\|\phi-\psi\| \|
\end{aligned}
$$

and also

$$
\|(g \phi)(t)-(\mathscr{g} \psi)(t)\|_{a_{2}} \leqq(1+|t|)^{-d_{2}} c_{2}^{\prime}\left(h_{1}, h_{2}, \eta\right)\|\phi-\psi\| .
$$

Hence from $\left(2^{\prime}\right),\left(3^{\prime}\right)$ and $\left(4^{\prime}\right)$ we obtain for $\phi, \psi \in X\left(\eta, \phi_{-}\right)$

$$
\|M \phi-M \psi\| \leqq\left\{\sum_{j=0}^{2} c_{j}^{\prime}\left(h_{1}, h_{2}, \eta\right)\right\}\|\phi-\psi\| \|
$$

Then we take $\eta$ so small that $\sum_{j=0}^{2} c_{j}^{\prime}\left(h_{1}, h_{2}, \eta\right)<1$ and fix an $\eta$, say $\eta_{0}$. Thus $M$ is a contraction mapping on $X\left(\eta, \phi_{-}\right)$for all positive $\eta \leqq \eta_{0}$. This guarantees existence of a unique fixed point $\phi$ in $X\left(\eta, \phi_{-}\right)$, which gives a global solution of (1) with $\|\phi\| \leqq 2 \eta_{0}$ by definition of $M$.

(II) Global uniqueness. Let $X\left(T, \alpha, \phi_{0}\right)$ be the set

$$
\left\{\phi \in C([0, T] ; \mathcal{H}) ; \phi(0)=\phi_{0}, \sup _{[0, T]}\left\|\phi(t)-e^{-i A t} \phi_{0}\right\| \leqq \alpha\right\},
$$

where $\alpha$ is an arbitrary fixed positive number. First we note that local uniqueness is true for the equation

$$
\phi(t)=e^{-i A t} \dot{\phi}_{0}+\int_{0}^{t} e^{-i A(t-s)} J(\phi(s)) d s
$$

in $X\left(T, \alpha, \phi_{0}\right)$, since, by virtue of $(\mathrm{H} 1),(\mathrm{H} 2)$ and $(\mathrm{H} 3)$,

$$
\begin{gathered}
\|\phi(t)-\psi(t)\| \leqq \int_{0}^{t} \| e^{-i A(t-s)}(J(\phi(s))-J(\phi(s)) \| d s \\
\leqq \sum_{j=1}^{2} h_{j} \sum_{\varepsilon_{j}} \int_{0}^{t}\left(\|\phi\|_{a_{j}}+\|\psi\|_{a_{j}}\right)^{p_{j}-\varepsilon_{j}}
\end{gathered}
$$




$$
\begin{aligned}
& \times\|\phi(s)-\phi(s)\|_{a_{j}}^{\varepsilon_{j}}\|\phi(s)-\phi(s)\|^{1-\varepsilon_{j}} d s \\
\leqq & \sum_{j=1}^{2} h_{j} \sum_{\varepsilon_{j}} \int_{0}^{t}(\|\phi\|+\|\phi\|)^{p_{j}-\varepsilon_{j}}\|\phi(s)-\psi(s)\| d s \text { for } t>0 .
\end{aligned}
$$

Now we shall prove global uniqueness. Let $\phi_{1}(t)$ be another solution of (1). Then by using $\left(2^{\prime}\right),\left(3^{\prime}\right)$ and $\left(4^{\prime}\right)$ we obtain the inequalities:

$$
\begin{aligned}
\| \phi(t)- & \phi_{1}(t) \| \leqq \sum_{j=1}^{2} h_{j}\left(\|\phi\|+\left\|\phi_{1}\right\|\right)^{p_{j}-\varepsilon_{j}} \\
& \times \int_{-\infty}^{t}(1+|s|)^{-d_{j} p_{j}} d s\left\|\phi-\phi_{1}\right\|
\end{aligned}
$$

$$
\begin{aligned}
& (1+|t|)^{d_{1}}\left\|\phi(t)-\phi_{1}(t)\right\|_{a_{1}} \\
& \leqq c \sum_{j=1}^{2} h_{j}\left(\sum_{\varepsilon_{j}}\left(\|\phi\|+\left\|\phi_{1}\right\| \mid\right)^{p_{j}-\varepsilon_{j}}+\sum_{w_{j}}\left(\|\phi\|+\left\|\phi_{1}\right\|\right)^{p_{j}-w_{j}}\right) \\
& \quad \times\left[\int_{-\infty}^{t}(1+|t|)^{d_{1}}(1+|t-s|)^{-d_{1}}(1+|s|)^{-d_{j} p_{j}} d s\right]\left\|\phi-\phi_{1}\right\|
\end{aligned}
$$

and

$$
\begin{aligned}
& (1+|t|)^{d_{2}}\left\|\phi(t)-\phi_{1}(t)\right\|_{a_{2}} \\
& \leqq c \sum_{j=1}^{2} h_{j}\left(\sum_{\varepsilon_{j}}\left(\|\phi\|+\left\|\phi_{1}\right\|\right)^{p_{j}-\varepsilon_{j}}+\sum_{z_{j}}\left(\|\phi\|+\left\|\phi_{1}\right\| \mid\right)^{p_{j-z_{j}}}\right) \\
& \quad \times\left[\int_{-\infty}^{t}(1+|t|)^{d_{2}}(1+|t-s|)^{-d_{2}}(1+|s|)^{-d_{j} p_{j}} d s\right]\left\|\phi-\phi_{1}\right\| .
\end{aligned}
$$

Hence follows

$$
\left\|\phi-\phi_{1}\right\| \leqq f(t)\left\|\phi-\phi_{1}\right\|
$$

from (5), (6), (7) for a continuous function $f(t)$. This function $f(t)$ tends to 0 as $t \rightarrow-\infty$ when $d_{1} p>d_{2}, d_{2} q>d_{1}$ and $p, q>1$, which is a consequence of Lemma 2.1 (b).

Combining (8) with local uniqueness yields the required uniqueness. Thus our proof is completed.

Our second main theorem is

Theorem 2.3 (the scattering operator for small data). Let all the hypotheses of Theorem 2.2 be satisfied and let $\phi(t)$ be the solution of (1) with Cauchy dat $\phi_{-} \in \Sigma_{\text {scat }}$ satisfying $\left\|\phi_{-}\right\|_{\text {scat }} \leqq \eta_{0}$. Then 
(a) for each $t, \phi(t) \in \Sigma_{\text {scat }}$ and

$$
\left\|\phi(t)-e^{-i t A} \phi_{-}\right\| \rightarrow 0 \text { as } t \rightarrow-\infty,
$$

(b) there exists $\phi_{+} \in \Sigma_{\text {scat }}$ with $\left\|\phi_{+}\right\|_{\text {scat }} \leqq 2 \eta_{0}$ such that

$$
\left\|\phi(t)-e^{-i t A} \phi_{+}\right\| \rightarrow 0 \quad \text { as } t \rightarrow+\infty
$$

and in case $p, q>1, d_{1} p>d_{2}, d_{2} q>d_{1}$

(c) $\left\|e^{i t A} \phi(t)-\phi_{ \pm}\right\|_{\mathrm{scat}} \rightarrow 0$ as $t \rightarrow \pm \infty$

(d) $S: \phi_{-} \rightarrow \phi_{+}$is an injective continuous map of the set $\left\{\psi \in \Sigma_{\text {scat }}\right.$; $\left.\|\psi\|_{\text {scat }} \leqq \eta_{0}\right\}$ into the set $\left\{\|\psi\|_{\text {scat }} \leqq 2 \eta_{0}\right\}$ in the $\|\cdot\|_{\text {scat }}$-topology.

Proof. (a) and a part of (c). To show $\left\|e^{-i r A} \phi(t)\right\|<+\infty$ for each $t$, we do estimations just as in $\left(2^{\prime}\right),\left(3^{\prime}\right),\left(4^{\prime}\right)$.

$$
\begin{aligned}
& \sup _{r \in \mathbb{R}}\left\|e^{-i r A} \phi(t)\right\| \leqq \sup _{r \in \mathbb{R}}\left\|e^{-i r A} e^{-i t A} \phi_{-}\right\|+\sup _{r \in \mathbb{R}} \int_{-\infty}^{t}\left\|e^{-i A(t-s+r)} J(\phi(s))\right\| d s \\
& \leqq \sup _{r \in \mathbb{R}}\left\|e^{-i r A} \phi_{-}\right\|+\sum_{j=1}^{2} h_{j}\left(2 \eta_{0}\right)^{p_{j}+1-\varepsilon_{j}} \int_{-\infty}^{t}(1+|s|)^{-d_{j} p_{j}} d s \\
& \sup _{r \in \mathbb{R}}(1+|r|)^{d_{1}}\left\|e^{-i r A} \phi(t)\right\|_{a_{1}} \leqq \sup _{r \in \mathbb{R}}(1+|r|)^{d_{1}}\left\|e^{-i A r} e^{-i \Delta t} \phi_{-}\right\|_{a_{1}} \\
& +\sup _{r \in \boldsymbol{R}}(1+|r|)^{d_{1}} \int_{-\infty}^{t}\left\|e^{-i A(t-s+r)} J(\phi(s))\right\|_{a_{1}} d s \\
& \leqq \sup _{r \in \boldsymbol{R}}(1+|r|)^{d_{1}}(1+|t+r|)^{-d_{1}}\left\|\phi_{-}\right\|_{\text {scat }} \\
& +c \sum_{j=1}^{2} h_{j}\left(2 \eta_{0}\right)^{p_{j}}\left[1+\left(2 \eta_{0}\right)^{1-\varepsilon_{j}}+\left(2 \eta_{0}\right)^{1-w_{j}}\right] \\
& \times \sup _{r \in \boldsymbol{R}}\left(1+\mid r_{1}\right)^{d_{1}} \int_{-\infty}^{t}(1+|t+r-s|)^{-d_{1}}(1+|s|)^{-d_{j} p_{j}} d s \\
& \sup _{r \in \mathbb{R}}(1+|r|)^{d_{2}}\left\|e^{-i r A} \phi(t)\right\|_{a_{2}} \leqq \sup _{r \in \mathbb{R}}(1+|r|)^{d_{2}}(1+|t+r|)^{-d_{2}}\left\|\phi_{-}\right\|_{\text {scat }} \\
& +c \sum_{j=1}^{2} h_{j}\left(2 \eta_{0}\right)^{p_{j}}\left[1+\left(2 \eta_{0}\right)^{1-\varepsilon_{j}}+\left(2 \eta_{0}\right)^{1-w_{j}}\right] \\
& \times \sup _{r \in \mathbb{R}}(1+|r|)^{d_{2}} \int_{-\infty}^{t}(1+|t+r-s|)^{-d_{2}}(1+|s|)^{-d_{j} p_{j}} d s .
\end{aligned}
$$

Thus, using Lemma 2.1, we conclude

$$
\|\phi(t)\|_{\text {scat }} \leqq c_{1}\left\|\phi_{-}\right\|_{\text {scat }}+c_{2}<\infty \text { for each } t
$$

where $c_{1}$ does not depend on $t$ but $c_{2}$ does. Similarly, when $d_{1} p_{1}>d_{2}$, 
$d_{2} p_{2}>d_{1}, p_{1}, p_{2}>1$

$$
\begin{aligned}
& \left\|e^{-i r A}\left(\phi(t)-e^{-i t A} \phi_{-}\right)\right\|=\left\|\phi(t)-e^{-i t A} \phi_{-}\right\| \\
& \leqq \int_{-\infty}^{t}\|J(\phi(s))\| d s \leqq \sum_{j=1}^{2} h_{j}\left(2 \eta_{0}\right)^{p_{j}+1-\varepsilon_{j}} \int_{-\infty}^{t}(1+|s|)^{-d_{j} p_{j}} d s \rightarrow 0 \\
& \quad(1+|r|)^{d_{1}}\left\|e^{-i A r}\left(\phi(t)-e^{-i t A} \phi_{-}\right)\right\|_{a_{1}} \\
& \leqq(1+|r|)^{d_{1}} \int_{-\infty}^{t}\left\|e^{-i A(r-s)} J(\phi(s))\right\|_{a_{1}} d s \\
& \leqq c(1+|r|)^{d_{1}} \sum_{j=1}^{2} \int_{-\infty}^{t}(1+|r-s|)^{-d_{1}}(1+|s|)^{-d_{j} p_{j}} d s \rightarrow 0 \text { as } t \rightarrow-\infty ;
\end{aligned}
$$

Just as above,

$$
(1+|r|)^{d_{2}}\left\|e^{-i A r}\left(\phi(t)-e^{-i t A} \phi_{-}\right)\right\|_{a_{2}} \rightarrow 0 \quad \text { as } t \rightarrow-\infty .
$$

Hence we proved

$$
\begin{aligned}
& \left\|\phi(t)-e^{-i t A} \phi_{-}\right\| \rightarrow 0 \\
& \left\|\phi(t)-e^{-i t A} \phi_{-}\right\|_{\mathrm{scat}} \rightarrow 0
\end{aligned}
$$

when $p_{1}, p_{2}>1, d_{1} p_{1}>d_{2}, d_{2} p_{2}>d_{1}$.

(b) Since $\|\phi\| \leqq 2 \eta_{0}$ by Theorem 2.2 and since for $t_{1}>t_{2}$

$$
\begin{aligned}
& \left\|e^{i t_{1} A} \phi\left(t_{1}\right)-e^{i t_{2} A} \phi\left(t_{2}\right)\right\| \leqq \int_{t_{1}}^{t_{2}}\left\|e^{i s A} J(\phi(s))\right\| d s \\
& \leqq \sum_{j=1}^{2} h_{j}\left(2 \eta_{0}\right)^{p_{j}+1-\varepsilon_{j}} \int_{t_{1}}^{t_{2}}(1+|s|)^{-d_{j} p_{j}} d s \rightarrow 0 \text { as } t_{1}, t_{2} \rightarrow \infty,
\end{aligned}
$$

there exists $\lim _{t \rightarrow \infty} e^{i t A} \phi_{-}=\phi_{+}$in $\mathcal{H}$, and

$$
\left\|e^{i t A} \phi(t)-\phi_{+}\right\|=\left\|\phi(t)-e^{-i t A} \phi_{+}\right\| \rightarrow 0
$$

as $t \rightarrow+\infty$. To show $\left\|\phi_{+}\right\|_{\text {scat }} \leqq 2 \eta_{0}$, letting $t \rightarrow+\infty$ (in the $\|\cdot\|$-topology) in the equation

$$
e^{i t A} \phi(t)=\phi_{-}+\int_{-\infty}^{t} e^{i s A} J(\phi(s)) d s
$$

we observe that

$$
\phi_{+}=\phi_{-}+\int_{-\infty}^{\infty} e^{i s A} J(\phi(s)) d s
$$


Then the same techniques as in (a) can be applied to $\left(^{*}\right)$ to obtain $\left\|\phi_{+}\right\|_{\text {scat }} \leqq 2 \eta_{0}$ for sufficiently small $\eta_{0}$.

Also replacing each $\left(e^{i t_{2} A} \phi\left(t_{2}\right)-e^{i t_{1} A} \phi\left(t_{1}\right)\right)$ and $\int_{t_{1}}^{t_{2}}$ for $(\phi(t)$ $\left.-e^{-i t A} \phi_{-}\right)$and $\int_{-\infty}^{t}$ respectively gives

$$
\lim _{t \rightarrow \infty}\left\|e^{i t A} \phi(t)-\phi_{+}\right\|_{\mathrm{scat}}=0
$$

Thus (b) and the remaining part of (c) are proved.

Finally we turn to prove (d). That $S$ is injective on $\left\{\|\psi\|_{\text {scat }} \leqq \eta_{0}\right\}$ can be derived in a similar way to the uniqueness proof in Theorem 2.2 as follows. Note that the solution $\phi(t)$ of (1) satisfies

$$
\phi(t)=e^{-i(t-\tau) A} \phi(\tau)+\int_{\tau}^{t} e^{-i(t-s) A} J(\phi(s)) d s .
$$

Let $\phi_{-}$and $\psi_{-}$correspond to $\phi_{+}$and let $\phi(t)$ and $\psi(t)$ be the corresponding solutions of (1) to Cauchy data $\phi_{-}$and $\psi_{-}$respectively. Then by (9) we have

$$
\phi(\tau)-\phi(\tau)=e^{i(t-\tau) A}(\phi(t)-\phi(t))-\int_{\tau}^{t} e^{-i(\tau-s) A}(J(\phi(s))-J(\phi(s))) d s .
$$

Estimations similar to (5), (6), (7) give

$$
\begin{aligned}
& \|\phi(\tau)-\phi(\tau)\| \leqq\|\phi(t)-\psi(t)\|+C(\phi, \phi)\|\phi-\psi\|_{[\tau, \infty)} \sum_{j} \int_{\tau}^{t}(1+|s|)^{-d_{j} p_{j}} d s, \\
& (1+|\tau|)^{d_{j}}\|\phi(\tau)-\psi(\tau)\|_{a_{j}} \leqq(1+|\tau|)^{d_{j}}\left\|e^{-i \tau A}\left(e^{-i t A} \phi(t)-e^{i t A} \psi(t)\right)\right\| \\
& +C(\phi, \phi)\|\phi-\psi\|_{[\tau, \infty)}(1+|\tau|)^{d_{j}} \int_{\tau}^{t}(1+|\tau-s|)^{-d_{j}}(1+|s|)^{-d_{j} p_{j}} d s
\end{aligned}
$$

from which follows

$$
\begin{aligned}
& \|\phi-\psi\|_{[\tau, \infty)} \leqq\left\|e^{i t A} \phi(t)-e^{i t A} \phi(t)\right\|_{\text {scat }} \\
& \quad+C(\phi, \phi)\|\phi-\psi\|_{[r, \infty)} \sum_{j}(1+|\tau|)^{d_{j}} \int_{\tau}^{t}(1+|\tau-s|)^{-d_{j}}(1+|s|)^{-d_{j} p_{j}} d s
\end{aligned}
$$

where $\quad\|\phi\|_{[\tau, \infty]}=\sup _{r \leq r<\infty}\left(\|\phi(r)\|+\sum_{j=1}^{2}(1+|r|)^{d_{j}}\|\phi(r)\|_{a_{j}}\right)$.

Then by Lemma $2.1(\mathrm{~b})$, for any $\varepsilon>0$ there exists $\tau_{0}>0$ such that

$$
\|\phi-\psi\|_{[r, \infty)} \leqq\left\|e^{i t A} \phi(t)-e^{i t A} \psi(t)\right\|_{s c a t}+\varepsilon\|\phi-\psi\|_{[r, \infty)}
$$

when $\tau \geqq \tau_{0}$. Letting $t \rightarrow \infty$, by (b) we have 


$$
\|\phi-\psi\|_{[\tau, \infty)} \leqq \varepsilon\|\phi-\psi\|_{[\tau, \infty)} \quad \text { for } \quad \tau \geqq \tau_{0}
$$

which is false unless $\|\phi-\psi\|_{[\tau, \infty)} \equiv 0\left(\tau \geqq \tau_{0}\right)$. Thus $\phi(t) \equiv \psi(t)$ for $\tau \geqq \tau_{0}$ Now given $\varepsilon>0$, choose $\delta>0$ so that

$$
\sup _{\tau_{0} \rightarrow \delta \leq s \leq \tau_{0}}\|\phi(s)-\psi(s)\|<\varepsilon .
$$

Then

$$
\begin{aligned}
0=\phi\left(\tau_{0}\right) & -\phi\left(\tau_{0}\right)=e^{-i A\left(\tau_{0}-\tau\right)}(\phi(\tau)-\psi(\tau)) \\
& +\int_{\tau}^{\tau_{0}} e^{-i A\left(\tau_{0}-s\right)}(J(\phi(s))-J(\phi(s))) d s .
\end{aligned}
$$

Hence if $\tau_{0}-\delta \leqq \tau \leqq \tau_{0}$,

$$
\|\phi(\tau)-\psi(\tau)\| \leqq \int_{\tau}^{\tau_{0}}\|J(\phi(s))-J(\phi(s))\| d s \leqq C \delta \sup _{\tau_{0}-\delta \leqq \tau \leqq \tau_{0}}\|\phi(\tau)-\psi(\tau)\|
$$

where $C$ depends only on $\eta$. Thus $\delta$ chosen so small as $C \delta<1$, the inequalities are false unless $\sup _{\tau_{0}-\delta \leq \tau \leq \tau_{0}}\|\phi(\tau)-\psi(\tau)\|=0$ which proves $\phi \equiv \psi$ when $\tau \geqq \tau_{0}-\delta$. Therefore $\phi \equiv \psi$ in $\boldsymbol{R}$ and so $\phi_{-}=\psi_{-}$.

To show the continuity of $S$ on $\left\{\|\psi\|_{\text {scat }} \leqq \eta_{0}\right\}$, we need estimate $\left\|\phi_{+}^{(1)}-\phi_{+}^{(2)}\right\|_{\text {scat }}$ where $\phi_{+}^{(i)}=S \phi_{-}^{(j)}$ for $j=1,2$. As is easily seen from estimates (2), (3), (4), it follows that

$$
\begin{aligned}
\left\|\phi_{1}(t)-\phi_{2}(t)\right\| \leqq\left\|\phi_{-}^{(1)}-\phi_{-}^{(2)}\right\| & +C\left(\eta_{0}\right)\left\|\phi_{1}-\phi_{2}\right\|, \\
(1+|t|)^{d_{j}}\left\|\phi_{1}(t)-\phi_{2}(t)\right\|_{a_{j}} \leqq & (1+|t|)^{d_{j}}\left\|e^{-i A t}\left(\phi_{-}^{(1)}-\phi_{-}^{(2)}\right)\right\|_{a_{j}} \\
& +C\left(\eta_{0}\right)\left\|\phi_{1}-\phi_{2}\right\| \quad \text { for } \quad j=1,2
\end{aligned}
$$

if $p, q>1, d_{1} p>d_{2}$ and $d_{2} q>d_{1}$, where $C\left(\eta_{0}\right) \rightarrow 0$ as $\left|\eta_{0}\right| \rightarrow 0$. Therefore we have

or

$$
\left\|\phi_{1}-\phi_{2}\right\| \leqq\left\|\phi_{-}^{(1)}-\phi_{-}^{(2)}\right\|_{\text {scat }}+3 C\left(\eta_{0}\right)\left\|\phi_{1}-\phi_{2}\right\|
$$

$$
\left\|\phi_{1}-\phi_{2}\right\| \leqq \frac{1}{1-3 C\left(\eta_{0}\right)}\left\|\phi_{-}^{(1)}-\phi_{-}^{(2)}\right\|_{\text {scat }} .
$$

Similarly

$$
\begin{aligned}
& (1+|r|)^{d_{j}}\left\|e^{-i A r}\left(e^{i A t} \phi_{1}(t)-e^{i A t} \phi_{2}(t)\right)\right\|_{a_{j}} \\
& \leqq(1+|r|)^{d_{j}}\left\|e^{-i A r}\left(\phi_{-}^{(1)}-\phi_{-}^{(2)}\right)\right\|_{a_{j}}+C\left(\eta_{0}\right)\left\|\phi_{1}-\phi_{2}\right\|
\end{aligned}
$$

when $p, q>1, d_{2} q>d_{1}$ and $d_{1} p>d_{2}$. Consequently 


$$
\begin{gathered}
\left\|e^{-i A t} \phi_{1}(t)-e^{i A t} \phi_{2}(t)\right\|_{\text {scat }} \leqq\left\|\phi_{-}^{(1)}-\phi_{-}^{(2)}\right\|_{\text {scat }}+3 C\left(\eta_{0}\right)\left\|\phi_{1}-\phi_{2}\right\| \\
\leqq \frac{1}{1-3 C\left(\eta_{0}\right)}\left\|\phi_{-}^{(1)}-\phi_{-}^{(2)}\right\|_{\text {scat }} \leqq 2\left\|\phi_{-}^{(1)}-\phi_{-}^{(2)}\right\|_{\text {scat }}
\end{gathered}
$$

if $\left|\eta_{0}\right|$ is taken so small as $3 C\left(\eta_{0}\right) \leqq 1 / 2$. Taking $t \rightarrow \infty$ we have

$$
\left\|\phi_{+}^{(1)}-\phi_{+}^{(2)}\right\|_{\text {scat }} \leqq 2\left\|\phi_{-}^{(1)}-\phi_{-}^{(2)}\right\|_{\text {scat }}
$$

for $\phi_{-}^{(j)} \in\left\{\|\phi\|_{\text {scat }} \leqq \eta_{0}\right\}$, which proves continuity of $S$.

Thus the proof has been completed.

\section{§3. Applications}

We consider a nonlinear Klein-Gordon equation

$$
\square u+m^{2} u+g_{1} u^{\beta}+g_{2} u_{t}^{r}=0, \quad x \in \mathbb{R}^{n}
$$

where $n=1$ or 3 , and $g_{1}, g_{2}$ are the coupling constants. Let

$$
A=i\left(\begin{array}{cc}
0 & I \\
-B^{2} & 0
\end{array}\right)
$$

where $B^{2}$ is the self-adjoint realization of $m^{2} I-\Delta$ in $L^{2}=L^{2}\left(\mathbb{R}^{n}\right)$, and let $J=J_{1}+J_{2}$ where

$$
J_{1}(\phi)=\left(\begin{array}{c}
0 \\
-g_{1} u^{\beta}
\end{array}\right), \quad J_{2}(\phi)=\left(\begin{array}{c}
0 \\
-g_{2} u_{t}^{r}
\end{array}\right) \quad \text { for } \quad \phi(t)=\left(\begin{array}{l}
u(\cdot, t) \\
u_{t}(\cdot, t)
\end{array}\right) .
$$

Then the equation (12) is written as

$$
\phi^{\prime}(t)=-i A \phi(t)+J(\phi(t))
$$

in its standard vector valued form. Further, this equation can be reformulated as an integral equation

$$
\phi(t)=e^{-i t A} \phi_{-}+\int_{-\infty}^{t} e^{-i A(t-s)} J(\phi(s)) d s
$$

provided that $\phi(t)$ has the Cauchy data $\phi_{-}$at $t=-\infty$. Now we define, for $n=3$, the solution space $\mathcal{H}$ by the completion of $D\left(B^{3}\right) \oplus D\left(B^{2}\right)$ with respect to the inner product

$$
(\phi, \psi)=\left(B^{3} u_{1}, B^{3} u_{2}\right)+\left(B^{2} v_{1}, B^{2} v_{2}\right)
$$

where $($,$) denotes the usual inner product in L^{2}$ and 


$$
\phi=\left(\begin{array}{l}
u_{1} \\
v_{1}
\end{array}\right), \quad \phi=\left(\begin{array}{l}
u_{2} \\
v_{2}
\end{array}\right) .
$$

Moreover we define the auxiliary norms $\|\phi\|_{a_{j}},\|\phi\|_{b_{j}}$ in $\mathcal{H}$ for $\phi=\left(\begin{array}{l}u \\ v\end{array}\right)$ $j=1,2$ by

$$
\begin{aligned}
& \|\phi\|_{a_{1}}=\|u\|_{\infty}, \quad\|\phi\|_{a_{2}}=\|v\|_{\infty} ;\|\phi\|_{b_{1}}=\left\|B^{3} u\right\|_{1}+\left\|B^{2} v\right\|_{1}, \\
& \|\phi\|_{b_{2}}=\left\|B^{3} u\right\|_{r}+\left\|B^{2} v\right\|_{r} \quad(1<r<2) .
\end{aligned}
$$

The following three lemmas are useful in our applications.

Lemma 3. $\mathbb{1}$ (Sobolev's inequality). Let $m$ and $n$ be positive numbers and let $p$ be defined by

$$
1 / p=1 / 2-m / n \text {. }
$$

Then for all $u \in C_{0}\left(\mathbb{R}^{n}\right)$, there is a constant $C$ such that

$$
\|u\|_{p} \leqq C\left\|B^{m} u\right\|_{2},
$$

where we take $p=\infty$ in case $m>n / 2$.

Lemma 3.2 (Nelson's theorem). Let $W_{t, a}(x), x \in \mathbb{R}^{n}$, be the functions whose Fourier transforms are

$$
\begin{aligned}
\widehat{W}_{t, a}(y) & =\left(m^{2}+y^{2}\right)^{-a} \exp \left[-i t\left(m^{2}+y^{2}\right)^{1 / 2}\right] \\
& =\widehat{E}_{t, a}-i \widehat{F}_{t, a}
\end{aligned}
$$

where $E_{t, a}=\left(m^{2}-\Delta\right)^{-a} \cos t\left(m^{2}-\Delta\right)^{1 / 2}$ and

$$
F_{t, a}=\left(m^{2}-\Delta\right)^{-a} \sin t\left(m^{2}-\Delta\right)^{1 / 2} \text {. }
$$

Fix $a>(n-1) / 4$ and $2 \leqq p<\infty$. Then for every $t \neq 0, W_{t, a}$ is in $L^{p}\left(\boldsymbol{R}^{n}\right)$ if and only if $2 a-(n+1) / 2>-1 / p$, and in this case

$$
\begin{aligned}
\left\|W_{t, a}\right\|_{p} & \simeq t^{n / p-n / 2} \text { if } s(p, a)>-2 \\
& \simeq(\log t)^{1 / p} t^{n / p-n / 2} \text { if } s(p, a)=-2, p>2 \\
& \simeq t^{(j-2 a)} t^{(n-2) / p} \text { if } s(p, a)<-2
\end{aligned}
$$

where $s(p, a)=p(2 a-(n+2) / 2)$ and $f(t) \simeq g(t)$ means that $f(t) / g(t)$ $=0(1)$ and $g(t) / f(t)=0(1)$ as $t \rightarrow \infty$. When $p=\infty$, for $t>0$

$$
\left\|W_{t, a}\right\|_{\infty} \simeq t^{-n / 2} \text { if } a \geqq(n+2) / 4
$$




$$
\simeq t^{1-2 a} \text { if }(n+1) / 4<a \leqq(n+2) / 4
$$

and

$$
\begin{aligned}
& W_{t, a}(|x|) \text { is unbounded along the cone }|x|=t>0 \text { if } \\
& (n+1) / 4 \geqq a>(n-1) / 4 \text {. }
\end{aligned}
$$

Lemma 3. 3 (Nirenberg's inequality). Let $D^{\alpha} f$ be the $\alpha$-th weak derivative of $f$ and let $\left|D^{i} f\right|_{p}=\max _{|\alpha|=i}\left\|D^{\alpha} f\right\|_{p}$. Suppose $f \in D\left(B^{a}\right)$ with $a \geqq 2$. Then

$$
\left|D^{i} f\right|_{p} \leqq \text { const. }\left|D^{k} f\right|_{2}^{r}\|f\|_{\infty}^{1-r}
$$

when $2 \leqq p<\infty, 2 \leqq k \leqq a, 0 \leqq j<k$, where $p^{-1}=j / n+r(1 / 2-k / n)$ for all with $j / k \leqq \gamma<1$.

Now in $\mathbb{R}^{3}$ we shall prove

Theorem 3.4. Let $\beta \geqq 3$ and $\gamma>7 / 2$. If the Cauchy data $\phi_{-}$at $t=-\infty$ is sufficiently small in the $\|\cdot\|_{\text {scat }}$-norm, then (12) has a unique global solution $\phi(t)=\left(\begin{array}{l}u(t) \\ u_{t}(t)\end{array}\right)$ and then there exists $\phi_{+}$with $\left\|\phi_{+}\right\|_{\text {scat }}$ small such that

$$
\left\|\phi(t)-e^{-i t A} \phi_{ \pm}\right\| \rightarrow 0,
$$

and moreover, except for $\beta=3$,

$$
(1+|t|)^{3 / 2}\left\|u(t)-\left[e^{-i t A} \phi_{ \pm}\right]_{1}\right\|_{\infty} \rightarrow 0
$$

and

$$
(1+|t|)^{d_{2}}\left\|u_{t}(t)-\left[e^{-i t A} \phi_{ \pm}\right]_{2}\right\|_{\infty} \rightarrow 0
$$

as $t \rightarrow \pm \infty$, where $[\phi]_{j}$ denotes the jth component of $\phi$ and $d_{2}$ is arbitrary but $3 / 2(\gamma-2)<d_{2}<1$.

Moreover the scattering operator $S: \phi_{-} \mapsto \phi_{+}$is injective and continuous in the $\|\cdot\|$-topology. Except for $\beta=3, S$ is continuous in the $\|\cdot\|_{\text {scat }}$-topology.

When $3 \leqq r \leqq 7 / 2$, also the same results are true for $d_{1}=3(\gamma-1)$ $/(3 r+2)$ and $d_{2}=1 / r$, where $1<r<2$.

Proof. From Lemma 3.1 (H1) follows immediately. To show that 
(H3) holds we estimate $\left\|B^{2}\left(u^{\alpha}-v^{\alpha}\right)\right\|_{r}$ for $1 \leqq r \leqq 2$ and $\alpha \geqq 3$. We denote $\partial f / \partial x_{i}$ simply by $f_{i}$. Since

$$
\begin{aligned}
B^{2}\left(u^{\alpha}-v^{\alpha}\right) & =\left(m^{2}-\Delta\right)\left(u^{\alpha}-v^{\alpha}\right) \\
& =m^{2}\left(u^{\alpha}-v^{\alpha}\right)-\sum_{j=1}^{3}\left(u^{\alpha}-v^{\alpha}\right)_{j j}
\end{aligned}
$$

and since

$$
\begin{aligned}
\left(u^{\alpha}-v^{\alpha}\right)_{j j}= & \alpha(\alpha-1)\left[u^{\alpha-2}\left(u_{j}^{2}-v_{j}^{2}\right)+v_{j}^{2}\left(u^{\alpha-2}-v^{\alpha-2}\right)\right] \\
& +\alpha\left[u^{\alpha-1}\left(u_{j j}-v_{j j}\right)+v_{j j}\left(u^{\alpha-1}-v^{\alpha-1}\right)\right] .
\end{aligned}
$$

Noting that $1 \leqq r \leqq 2$ and $\beta \geqq 3$ we obtain

$$
\left\|B^{2}\left(u^{\alpha}-v^{\alpha}\right)\right\|_{r} \leqq g \sum_{\varepsilon}\left(\|u\|_{\infty}+\|v\|_{\infty}\right)^{\alpha-2 / r-\varepsilon}\|u-v\|_{\infty}^{\varepsilon}\left\|B^{2}(u-v)\right\|^{1-\varepsilon}
$$

where $\varepsilon$ runs through $F_{r}=\{0,1 / 2,2-2 / r\}$ and $g=g\left(\left\|B^{2} u\right\|_{2},\left\|B^{2} v\right\|_{2}\right)$ with $\lim _{a, b \rightarrow 0} g(a, b)=0$. In the hypothesis (H3) if we choose $F_{1}$ as the finite set through which both $\varepsilon_{1}=\varepsilon_{2}(=\varepsilon)$ run, then we have

$$
\begin{aligned}
& \left\|J_{j}(\phi)-J_{j}(\psi)\right\| \\
& \quad \leqq h_{j} \sum_{\varepsilon}\left(\|\phi\|_{a_{j}}+\|\psi\|_{a_{j}}\right)^{\alpha-1-\varepsilon}\|\phi-\psi\|_{a_{j}}^{\varepsilon}\|\phi-\psi\|^{1-\varepsilon} .
\end{aligned}
$$

From (H1) and (13) it follows immediately that

$$
\left\|J_{j}(\phi)-J_{j}(\psi)\right\| \leqq h_{j}^{\prime} \sum_{\varepsilon}\left(\|\phi\|_{a_{j}}+\|\psi\|_{a_{j}}\right)^{\alpha-2}\|\phi-\psi\|
$$

where $h_{j}^{\prime}=c h_{j}$. Also we have

$$
\begin{aligned}
& \left\|J_{j}(\phi)-J_{j}(\psi)\right\|_{b_{1}} \\
& \quad \leqq h_{j} \sum_{\sigma}\left(\|\phi\|_{a_{j}}+\|\phi\|_{a_{j}}\right)^{\alpha-2-\sigma}\|\phi-\psi\|_{a_{j}}^{\sigma}\|\phi-\psi\|^{1-\sigma}
\end{aligned}
$$

and

$$
\begin{aligned}
& \left\|J_{j}(\phi)-J_{j}(\phi)\right\|_{b_{2}} \\
& \quad \leqq h_{j} \sum_{\rho}\left(\|\phi\|_{a_{j}}+\|\psi\|_{a_{j}}\right)^{\alpha-2 / r-\rho}\|\phi-\psi\|_{a_{j}}^{\rho}\|\phi-\psi\|^{1-\rho}
\end{aligned}
$$

where $\sigma$ runs through $F_{2}, \rho$ through $F_{r}$ and $\alpha$ equals $\beta$ or $\gamma$ according as $j=1$ or 2 .

Now, although (H2) cannot be verified to our example, we proceed the theorem directly as in Theorems $2.2,2.3$, with the help of (H1), 
(H3) just obtained above. Thus we define as in the proof of Theorem 2.2

$$
X\left(\eta, \phi_{-}\right)=\left\{\psi \in C(R ; \mathcal{H}) ;\left\|\psi(t)-e^{-i t A} \phi_{-}\right\| \leqq \eta,\left\|\phi_{-}\right\|_{\text {scat }} \leqq \eta\right\}
$$

and

$$
(M \phi)(t)=e^{-i t A} \phi_{-}+\int_{-\infty}^{t} e^{-i(t-s) d}\left(J_{1}(\phi(s))+J_{2}(\phi(s))\right) d s .
$$

By (13), (14), (15) and Lemma 3.2, the following estimates are immediate: for $\phi, \psi \in X\left(\eta, \phi_{-}\right)$

$$
\begin{aligned}
& \|M(\phi)-M(\psi)\| \\
& \leqq \sum_{\varepsilon}\left[h_{1} \int_{-\infty}^{t}\left(\|\phi(s)\|_{a_{1}}+\|\psi(s)\|_{a_{1}}\right)^{\beta-1-\varepsilon}\|\phi(s)-\psi(s)\|_{a_{1}}^{\varepsilon}\|\phi(s)-\psi(s)\|^{1-\varepsilon} d s\right. \\
& \left.\quad+h_{2} \int_{-\infty}^{i}\left(\|\phi(s)\|_{a_{2}}+\|\phi(s)\|_{a_{2}}\right)^{\gamma-1-\varepsilon}\|\phi(s)-\psi(s)\|_{a_{2}}^{\varepsilon}\|\phi(s)-\psi(s)\|^{1-\varepsilon} d s\right] \\
& \leqq \sum_{\varepsilon}\left[h_{1}(4 \eta)^{\beta-1-\varepsilon} \int_{-\infty}^{\infty}(1+|s|)^{(\beta-1) d_{1}} d s\right. \\
& \left.\quad+h_{2}(4 \eta)^{r-1-\varepsilon} \int_{-\infty}^{\infty}(1+|s|)^{(\gamma-1) d_{2}} d s\right]\|\phi-\psi\| .
\end{aligned}
$$

The integrals in the third member are convergent provided that

$$
(\beta-1) d_{1}>1, \quad(\gamma-1) d_{2}>1 .
$$

Since

$$
e^{-i A t}=\left(\begin{array}{cc}
\cos t B & B^{-1} \sin t B \\
-B \sin t B & \cos t B
\end{array}\right)
$$

and so since

$$
M(\phi)(t)-M(\phi)(t)=-\int_{-\infty}^{t}\left(\begin{array}{r}
B^{-1} \sin (t-s) B\left(g_{1} u^{\beta}+g_{2} u_{s}^{r}\right) \\
\cos (t-s) B\left(g_{1} u^{\beta}+g_{2} u_{s}^{r}\right)
\end{array}\right) d s,
$$

it follows from the definitions of auxiliary norms that

$$
\begin{aligned}
\|M(\phi)-M(\psi)\|_{a_{1}} & \leqq\left|g_{1}\right| \int_{-\infty}^{t} \sup _{x}\left|B^{-1} \sin (t-s) B\left(u^{\beta}-v^{\beta}\right)\right| d s \\
& +\left|g_{2}\right| \int_{-\infty}^{t} \sup _{x}\left|B^{-1} \sin (t-s) B\left(u_{s}^{r}-v_{s}^{r}\right)\right| d s \\
& \leqq\left|g_{1}\right| \int_{-\infty}^{t} \sup _{x}\left|\mathcal{E}^{-1}\left[\widehat{F}_{t-s, 3} \cdot B^{2}\left(u^{\beta}-v^{\beta}\right)\right]\right| d s
\end{aligned}
$$




$$
\begin{aligned}
& +\left|g_{2}\right| \int_{-\infty}^{t} \sup _{x}\left|\mathscr{F}^{-1}\left[\widehat{F}_{t-s, 3} \cdot B^{2}\left(u_{s}^{r}-v_{s}^{r}\right)\right]\right| d s \\
& \leqq\left|g_{1}\right| \int_{-\infty}^{t} \sup _{x}\left|F_{t-s, 3} * B^{2}\left(u^{\beta}-v^{\beta}\right)\right| d s \\
& +\left|g_{2}\right| \int_{-\infty}^{t} \sup _{x}\left|F_{t-s, 3} * B^{2}\left(u_{s}^{r}-v_{s}^{r}\right)\right| d s \\
& \leqq\left|g_{1}\right| \int_{-\infty}^{t}\left\|F_{t-s, 3}\right\|_{p /(p-1)}\left\|B^{2}\left(u^{\beta}-v^{\beta}\right)\right\|_{p} d s \\
& +\left|g_{2}\right| \int_{-\infty}^{t}\left\|F_{t-s, 3}\right\|_{q /(q-1)}\left\|B^{2}\left(u u_{s}^{r}-v_{s}^{r}\right)\right\|_{q} d s
\end{aligned}
$$

Now applying Lemma 3.2 to the last members of above inequalities, we obtain

$$
\begin{aligned}
& \|M(\phi)-M(\psi)\|_{a_{1}} \leqq\|\phi-\psi\| \| \\
& \quad \times\left\{c \sum_{\sigma \in F_{p}}\left|g_{1}\right|(4 \eta)^{r-2 / p-\sigma} \int_{-\infty}^{t}(1+|t-s|)^{-(3 / p-3 / 2)}(1+|s|)^{-(\beta-2 / p) d_{1}} d s\right. \\
& \left.\quad+c \sum_{\sigma^{\prime} \in F_{q}}\left|g_{2}\right|(4 \eta)^{r-2 / q-\sigma^{\prime}} \int_{-\infty}^{t}(1+|t-s|)^{-(3 / q-3 / 2)}(1+|s|)^{-(r-2 / q) d_{2}} d s\right\} .
\end{aligned}
$$

Taking Lemma 2.1 into consideration, we impose the following conditions to the exponents appearing in the above integrals:

$$
\begin{aligned}
& \max \left(3 / p-3 / 2,(\beta-2 / p) d_{1}\right)>1 \\
& \min \left(3 / p-3 / 2,(\beta-2 / p) d_{1}\right) \geqq d_{1} \\
& \max \left(3 / q-3 / 2,(\gamma-2 / q) d_{2}\right)>1, \\
& \min \left(3 / q-3 / 2,(\gamma-2 / q) d_{2}\right) \geqq d_{1}
\end{aligned}
$$

where $1 \leqq p, q<2$.

Similarly we have

$$
\begin{aligned}
\|M(\phi)-M(\phi)\|_{a_{2}} & \leqq \int_{-\infty}^{t}\left\|E_{t-s, 2}\right\|_{r /(r-1)}\left\|B^{2}\left(u^{\beta}-v^{\beta}\right)\right\|_{r} d s \\
& +\int_{-\infty}^{t}\left\|E_{t-s, 2}\right\|_{r /(r-1)}\left\|B^{2}\left(u_{s}^{r}-v_{s}^{r}\right)\right\|_{r} d s,
\end{aligned}
$$

and so

$$
\begin{aligned}
& \|M(\phi)-M(\psi)\|_{a_{2}} \leqq\|\phi-\psi\| \\
& \quad \times c \sum_{\rho \in \vec{F}_{r}}\left\{\left|g_{1}\right|(4 \eta)^{\beta-2 / r-\rho} \int_{-\infty}^{t}(1+|t-s|)^{-1 / r}(1+|s|)^{-(\beta-2 / r) d_{1}} d s\right.
\end{aligned}
$$




$$
\left.+\left|g_{2}\right|(4 \eta)^{r-2 / r-\rho} \int_{-\infty}^{\iota}(1+|t-s|)^{-1 / r}(1+|s|)^{-(r-2 / r) d_{2}} d s\right\}
$$

with the conditions

$$
\begin{aligned}
& \max \left(1 / r,(\beta-2 / r) d_{1}\right)>1 \\
& \min \left(1 / r,(\beta-2 / r) d_{1}\right) \geqq d_{2} \\
& \max \left(1 / r,(\gamma-2 / r) d_{2}\right)>1 \\
& \min \left(1 / r,(\gamma-2 / r) d_{2}\right) \geqq d_{2}
\end{aligned}
$$

where $1<r<2$.

For $\beta \geqq 3$ and $\gamma>7 / 2$ we may choose $p=1, q=1$ and $r$ arbitrary but $1<r<2$ so that (16) (20) hold, whence $d_{1}=3 / 2$ and $d_{2}$ arbitrary but $1 / 2<d_{2}<1$. For $\beta \geqq 3$ and $7 / 2 \geqq \gamma \geqq 3$ we may choose $p=1, d_{2}=1 / r$ with $1<r<4 / 3$ so that (16), (17), (19) and (20) hold, provided $d_{1}>1$. Actually (20) follows from $r \geqq 3>r+2 / r$ when $1<r<4 / 3$. Concerning (18) we choose $q$ with

$$
3 / q-3 / 2=(\gamma-2 / q) \cdot 1 / r
$$

which implies $3 / q-3 / 2=3(r-1) /(3 r+2)$. Since $3 / q-3 / 2>1$ when $1<r<4 / 3$, we thus obtain

$$
d_{1}=3(\gamma-1) /(3 r+2)<3(\gamma-1) / 5 .
$$

Consequently we have proved by Theorems 2.2 and 2.3 the existence of a global unique solution and of scattering operator for small data. We finally note that continuity of $S$ in $\|\cdot\|$-topology is derived from (13') just as in $[8]$.

Q.E.D.

In case $n=1$ we define $\mathcal{H}$ by completion of $D\left(B^{2}\right) \oplus D\left(B^{1}\right)$ with respect to the inner product

$$
(\phi, \psi)=\left(B^{2} u_{1}, B^{2} u_{2}\right)+\left(B v_{1}, B v_{2}\right) .
$$

Then the corresponding conditions to $(16) \sim(20)$,

$$
\begin{aligned}
& (\beta-1) d_{1}>1,(\gamma-1) d_{2}>1 \\
& \max \left(1 / p-1 / 2,(\beta-2 / p) d_{1}\right)>1 \\
& \min \left(1 / p-1 / 2,(\beta-2 / p) d_{1}\right) \geqq d_{1}
\end{aligned}
$$




$$
\begin{aligned}
& \max \left(1 / q-1 / 2,(\gamma-2 / q) d_{2}\right)>1 \\
& \min \left(1 / q-1 / 2,(\gamma-2 / q) d_{2}\right) \geqq d_{1} \\
& \max \left(1 / r-1 / 2,(\beta-2 / r) d_{1}\right)>1 \\
& \min \left(1 / r-1 / 2,(\beta-2 / r) d_{1}\right) \geqq d_{2}
\end{aligned}
$$

and

$$
\begin{aligned}
& \max \left(1 / r-1 / 2,(\gamma-2 / r) d_{2}\right)>1 \\
& \min \left(1 / r-1 / 2,(\gamma-2 / r) d_{2}\right) \geqq d_{2} .
\end{aligned}
$$

should be imposed where $1 \leqq p, q \leqq 2$ and $4 / 3<r \leqq 2$.

Then we have analogously to the proof of Theorem 3.4.

Theorem 3. 5. The same claims as Theorem 3. 4 hold with $d_{1}$ $=1 / 2, d_{2}<1 / 4$ for $\beta>4, \gamma>6$.

For $11 / 2<\gamma \leqq 6, \beta$ should be chosen as $\beta>\gamma-4+2 /(\gamma-5)$ with $d_{1}<(\gamma-5) / 2, d_{2}<1 / 4$.

\section{References}

[1] Chadam, J. M., Asymptotics for $\square u=m^{2} u+G\left(x, t, u, u_{x}, u_{t}\right)$, I. Global existence and decay, Ann. Scuola. Norm. Sup. Pisa. 26 (1972), 33-65.

[2] Asymptotics for $\square u=m^{2} u+G\left(x, t, u, u_{x}, u_{t}\right)$, II. Scattering theory, Ann. Scuola. Norm. Sup. Pisa. 26 (1972), 67-95.

[3] Lions, J. L., Problemes aux limites dans les equations aux derivees partielles, University of Montreal Press, 1966.

[4] Morawetz, C., Notes on Time Decay and Scattering for some Hyperbolic Problems, Society for Industrial and Applied Mathematics, Philadelphia, 1975.

[5] Morawetz, C. and W. Strauss, Decay and scattering of solutions of a nonlinear relativistic wave equation, Comm. Pure Appl. Math. 25 (1972), 1-31.

[6] Nelson. S., On some solutions to the Klein-Gordon equation related to an integral of Sonine, Trans. AMS. 154 (1971), 227-237.

[7] Nirenberg, L., On elliptic partial differential equations, Ann. Scuola. Norm. Sup. Pisa 13 (1959), 115-162.

[8] Reed, M., Abstract non-linear wave equations, Springer-Verlag 507, 1976.

[9] Segal, I., Non-linear semi-groups, Ann. Math. 78 (1963), 339-364.

[10] Dispersion for nonlinear relativistic equation, II, Ann. Scient. Ec. Norm. Sup. ser 4, t. 1 (1968), 459-497.

[11] Strauss, W., Decay and asymptotics for $\square u=F(u)$, J. Func. Anal. 2(1968), 409-457.

[12] - - - , Nonlinear scattering theory, in Scattering Theory in Mathematical Physics, ed. J. A. Lavita and J.-P. Merchand, Reide Pub. Holland, (1974), 53-78. 